\title{
A case of secondary somatosensory epilepsy with a left deep parietal opercular lesion: successful tumor resection using a transsubcentral gyral approach during awake surgery
}

\author{
Satoshi Maesawa, MD, PhD, ${ }^{1,2}$ Masazumi Fujii, MD, PhD, ${ }^{1}$ Miyako Futamura, ST, BAPsy, ${ }^{3}$ \\ Yuichiro Hayashi, PhD, ${ }^{4}$ Kentaro lijima, MD, ${ }^{1}$ and Toshihiko Wakabayashi, MD, PhD ${ }^{1}$ \\ 'Department of Neurosurgery, Nagoya University Graduate School of Medicine; ${ }^{2 B}$ rain and Mind Research Center, Nagoya \\ University; ${ }^{3}$ Department of Rehabilitation, National Hospital Organization, Nagoya Medical Center; and ${ }^{4}$ Information and \\ Communications Headquarters, Nagoya University, Nagoya, Japan
}

\begin{abstract}
Few studies have examined the clinical characteristics of patients with lesions in the deep parietal operculum facing the sylvian fissure, the region recognized as the secondary somatosensory area (SII). Moreover, surgical approaches in this region are challenging. In this paper the authors report on a patient presenting with SIl epilepsy with a tumor in the left deep parietal operculum. The patient was a 24-year-old man who suffered daily partial seizures with extremely uncomfortable dysesthesia and/or occasional pain on his right side. MRI revealed a tumor in the medial aspect of the anterior transverse parietal gyrus, surrounding the posterior insular point. Long-term video electroencephalography monitoring with scalp electrodes failed to show relevant changes to seizures. Resection with cortical and subcortical mapping under awake conditions was performed. A negative response to stimulation was observed at the subcentral gyrus during language and somatosensory tasks; thus, the transcortical approach (specifically, a transsubcentral gyral approach) was used through this region. Subcortical stimulation at the medial aspect of the anterior parietal gyrus and the posterior insula around the posterior insular point elicited strong dysesthesia and pain in his right side, similar to manifestation of his seizure. The tumor was completely removed and pathologically diagnosed as pleomorphic xanthoastrocytoma. His epilepsy disappeared without neurological deterioration postoperatively. In this case study, 3 points are clinically significant. First, the clinical manifestation of this case was quite rare, although still representative of SIl epilepsy. Second, the location of the lesion made surgical removal challenging, and the transsubcentral gyral approach was useful when intraoperative mapping was performed during awake surgery. Third, intraoperative mapping demonstrated that the patient experienced pain with electrical stimulation around the posterior insular point. Thus, this report demonstrated the safe and effective use of the transsubcentral gyral approach during awake surgery to resect deep parietal opercular lesions, clarified electrophysiological characteristics in the SII area, and achieved successful tumor resection with good control of epilepsy.
\end{abstract}

http://thejns.org/doi/abs/10.3171/2015.2.JNS142737

KEY WORDS secondary somatosensory epilepsy; parietal operculum; awake surgery; subcentral gyrus; diagnostic and operative techniques

$\mathrm{T}$ HE human secondary somatosensory cortex (SII) was reported by Penfield and Jasper in 1954 through research on brain mapping with corticoelectrical stimulation. ${ }^{24}$ The SII is functionally located in the parietal operculum on the ceiling of the sylvian fissure. This area has been recently examined by functional imaging methods, such as functional MRI and magnetoencephalogra- phy, and its functional role has been determined. . $^{3,411,12,15,26}$ The contralateral primary somatosensory cortex processes and encodes the characteristics and intensities of the sensory inputs, whereas the bilateral SII is assumed to perform higher order functions, including somatosensory integration, integration of information from 2 body halves, attention, learning, and memory. ${ }^{2,3,5}$ Moreover, stimulation

ABBREVIATIONS EEG = electroencephalography; SII = secondary somatosensory cortex; SLTA = Standard Language Test of Aphasia; WAIS-III = Wechsler Adult Intelligence Scale-III; WMS-R = Wechsler Memory Scale-R.

SUBMITTED December 2, 2014. ACCEPTED February 3, 2015.

INCLUDE WHEN CITING Published online August 21, 2015; DOI: 10.3171/2015.2.JNS142737. 
of the SII region causes tingling/pricking sensations and even robust pain. ${ }^{20}$ Although several electrophysiological, anatomical, and imaging studies of the SII area have been performed, the clinical relevance of these methods in humans has not been fully elucidated.

In this report, we describe a patient with a lesion in the left deep parietal operculum, presenting with medically uncontrollable seizures, dysesthesia in the diffuse area of the body, and occasional pain, which was mostly caused by seizures related to the SII area. We performed tumor removal by cortical and subcortical mapping under awake surgery through the subcentral gyral approach, specifically a transsubcentral gyral approach. This method involved making a corridor in the subcentral gyrus for approaching this lesion after confirmation of negative mapping. Subcortical stimulation of this region resulted in pain. This is the first report that describes the clinical characteristics and surgical treatment of a patient with a lesion in the deep parietal operculum around the posterior insular point in detail. We would like to stress 3 points: 1) lesions in the deep parietal operculum could cause SII epilepsy; 2) surgical approaches for resection of this lesion were challenging, and the transsubcentral gyral approach was safe and advantageous; and 3) lesions within the SII region cause pain with direct electrical stimulation.

\section{Case Report}

Preoperative Evaluation

A 24-year-old man presented at our hospital with a 7 -year history of medically refractory daily seizures. He was otherwise free from neurological symptoms. His seizures were partial seizures, manifesting extremely uncomfortable dysesthesia and occasional pain in diffuse areas in his right side. Somatotopic distribution was unclear, and he complained that a pathological sensation (uncomfortable dysesthesia and occasional pain) frequently occurred in his right arm and in his face, but occasionally spread to the lower limb, head, or even to the contralateral body. The seizures occurred approximately 5-10 times per day, despite the patient taking $400 \mathrm{mg}$ of carbamazepine, 200 $\mathrm{mg}$ of lamotrigine, and $2000 \mathrm{mg}$ of levetiracetam daily. $\mathrm{He}$ also experienced generalized seizures approximately once per year. His Karnofsky performance status was 90. He was right handed, as assessed by the Edinburgh Inventory. Left-side dominance for language was confirmed by functional MRI using verb generation and picture naming tasks. Cognitive function, including language, was evaluated using the Standard Language Test of Aphasia (SLTA; Japan Society for Higher Brain Dysfunction, SLTA committee, 1977), ${ }^{30}$ Wechsler Adult Intelligence Scale-III (WAIS-III), Wechsler Memory Scale-R (WMS$\mathrm{R}$ ), and Clinical Assessment for Attention, which revealed no cognitive dysfunction or speech disturbance (Table 1).

In imaging studies, MRI revealed a lesion of $20 \mathrm{~mm}$ in diameter around the posterior insular point, which showed high signal intensity on T2-weighted and FLAIR images (Fig. 1B and D), and a well-circumscribed, very low-intensity lesion on STIR images corresponding to calcification (Fig. 1E). T1-weighted images showed isointensity and obscure signals, but the lesion was slightly enhanced by $\mathrm{Gd}$
TABLE 1. Pre- and postoperative results of neuropsychological tests

\begin{tabular}{|c|c|c|}
\hline Neuropsychological Test & Preop & Postop \\
\hline \multicolumn{3}{|l|}{ SLTA } \\
\hline Auditory comprehension & $10 / 10$ & $10 / 10$ \\
\hline Naming & $20 / 20$ & $20 / 20$ \\
\hline Sentence repetition & $5 / 5$ & $5 / 5$ \\
\hline Reading aloud short sentences & $5 / 5$ & $5 / 5$ \\
\hline Reading comprehension & $10 / 10$ & $10 / 10$ \\
\hline Dictation of Kana letters & $10 / 10$ & $10 / 10$ \\
\hline Dictation of short sentences & $5 / 5$ & $5 / 5$ \\
\hline \multicolumn{3}{|l|}{ WAIS-III } \\
\hline Verbal IQ & 118 & 116 \\
\hline Performance IQ & 117 & 113 \\
\hline Full IQ & 120 & 116 \\
\hline Verbal comprehension & 109 & 100 \\
\hline Perceptual organization & 121 & 121 \\
\hline Working memory & 135 & 130 \\
\hline Performance speed & 100 & 100 \\
\hline \multicolumn{3}{|l|}{ WMS-R } \\
\hline Verbal memory & 110 & 123 \\
\hline Visual memory & 116 & 116 \\
\hline Generalized memory & 113 & 124 \\
\hline Attention/concentration & 128 & 137 \\
\hline Delayed recall & 105 & 114 \\
\hline
\end{tabular}

$\mathrm{IQ}=$ Intelligence quotient.

(Fig. 1A). FDG-PET showed relatively low uptake in the lesion. Sagittal and coronal T2-weighted images revealed that the lesion was located outside of the insula but adjacent to the posterior insular point, i.e., in the deep parietal operculum (Fig. 1C and F), anatomically corresponding to the anterior transverse parietal gyrus (Fig. 2) ${ }^{31}$ Preoperative differential diagnosis included cavernous malformation, or Grade I-III gliomas (oligodendroglioma, pleomorphic xanthoastrocytoma, or ganglioglioma). Long-term video electroencephalography (EEG) monitoring was performed for determination of epileptogenesis. In the interictal phase, small spikes were sporadically observed in the left frontal (F3) or temporal areas (T3). We captured more than 10 partial seizures during recording, but EEG changes did not correspond to the seizures. We suspected that the seizures might start at a deep location within the hemisphere, making them difficult to record superficially using scalp electrodes. Thus, we recommended examination with intracerebral depth electrodes; however, the patient refused this procedure due to the risk of intracranial hemorrhage, possibly caused by insertion of the depth electrode, and due to possible damage of the cortex in the language-dominant side. Tumor resection was then performed, using awake surgery to preserve language function. The patient understood the potential complications and risks of this procedure and agreed to this treatment, providing written informed consent. Surgical procedures in addition to awake craniotomy, including intraoperative electrical mapping, were approved by the ethical commit- 

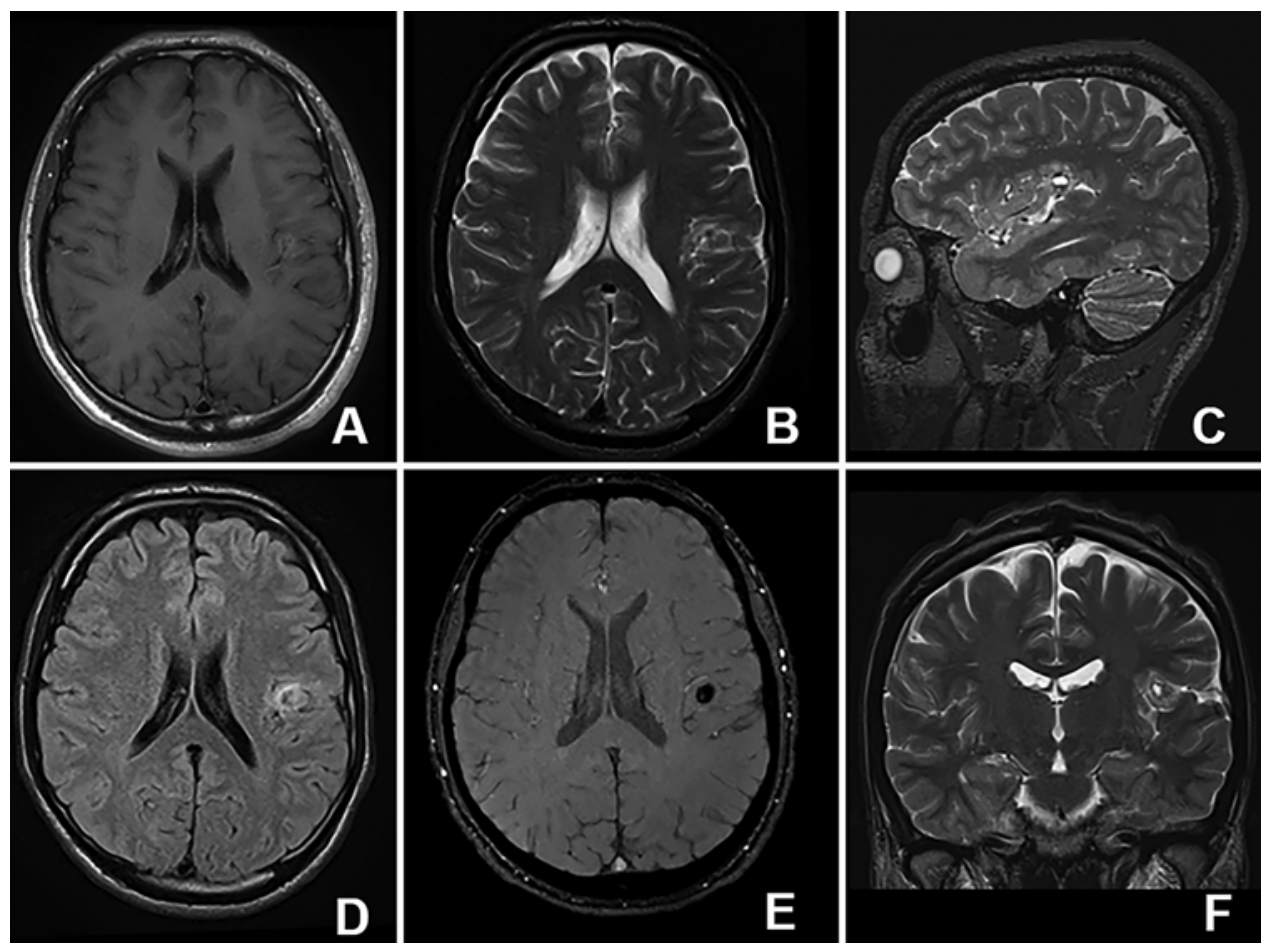

FIG. 1. Preoperative MR images. A: Axial T1-weighted image with Gd. B: Axial T2-weighted image. C: Sagittal T2-weighted image. D: Axial FLAIR image. E: Axial STIR image. F: Coronal T2-weighted image. A small lesion was located in the left anterior transverse parietal gyrus.

tee of Nagoya University Graduate School of Medicine and Nagoya University Hospital.

\section{Surgical Approach}

The lesion was located in the anterior transverse gyrus, the deep medial part of the parietal operculum covering the posterior insular point. To approach this lesion, opening the sylvian fissure widely within this region was challenging because of the complexity of the vasculature. Even if the sylvian fissure was opened, because the posterior insular point was located posteriorly and rostrally from the opening area, it was difficult to sufficiently secure the operative field to remove the tumor. Accordingly, we chose the transcortical approach via the subcentral gyrus located in the region inferior to the precentral and postcentral gyri (i.e., the transsubcentral gyral approach; Fig. 2).

\section{Operation (Intraoperative Mapping and Tumor Removal)}

The awake surgery was performed following the guidelines issued by the Guideline Committee of the Japanese Awake Surgery Conference in 2012, ${ }^{16}$ and our techniques for mapping were generally described in a previous report. ${ }^{10,18}$ General anesthesia was induced with a laryngeal mask (i-gel, Intersurgical), followed by a wide frontotemporal craniotomy, which exposed the sylvian fissure and the frontal and parietal operculum (including the triangular part of the inferior frontal gyrus anteriorly, the angular gyrus posteriorly, the middle frontal gyrus superiorly, and the middle temporal gyrus inferiorly). The tumor location and anatomical landmarks were verified using BrainLAB (Vector Vision Compact). The sylvian fissure was gently opened to the posterior end of the fissure, preserving the bridging veins; therefore, the posterior side of the sylvian fissure was not widely opened. The patient was then awakened, and the laryngeal mask was removed.

Cortical language mapping was performed during the awake portion of the surgery. A bipolar electrode (Unique Medical) with 5-mm tip spacing was used to apply electrical stimulation with a biphasic current intensity between 2 and $6 \mathrm{~mA}(60-\mathrm{Hz}$ pulse frequency, $0.5-\mathrm{msec}$ single-pulse phase, 8-second tissue contact; Neuromaster MEE1200, Nihon Kohden). Electrocorticograms were recorded during brain mapping and tumor removal with 3 strip electrodes (18 contacts). First, we identified the motor cortex (the precentral gyrus [tags 3 and 4 in Fig. 3B and C]) and somatosensory cortex (the posterior central gyrus [tags 1 and 2 in Fig. 3B and C]) by confirming moving disturbance and dysesthesia, respectively. Next, we evaluated language function using the object naming and auditory comprehension tasks. Aside from 1 site without stimulation (as a control), each site was tested 3 times with stimulation. ${ }^{23}$ The Broca area (the triangular gyrus [tags 6 and 7] and the opercular gyrus [tag 5] in Fig. 3B) and Wernicke area (the superior temporal gyrus [tags 9 and 10 in Fig. 3B]) were identified by anomia, speech arrest, or slowness during object naming and auditory comprehension language tasks. After determining the appropriate cortical areas, the parietal operculum (the posterior central gyrus) was gently retracted, and the subcentral gyrus, just medial and inferior to the sylvian veins, was exposed (Fig. 3C). We stimulated the surface of the subcentral gyrus with 4-6 $\mathrm{mA}$ (tag 11 in Fig. 3A); however, no positive response was observed in terms of motor or sensory function. The patient did not feel any sensation throughout his whole body, 


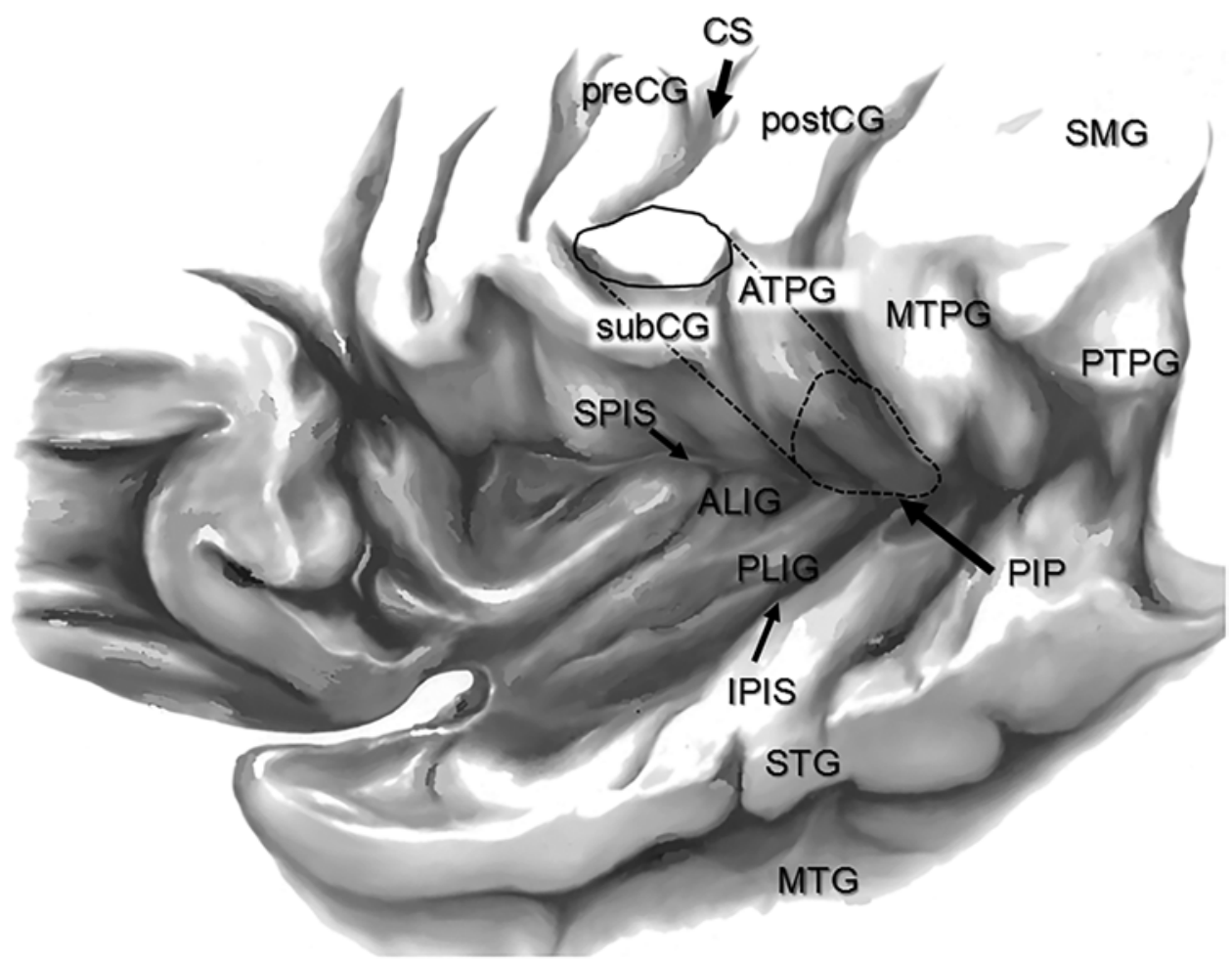

FIG. 2. A schematic diagram showing the anatomy of the parietal operculum and the transcortical approach via the subcentral gyrus (i.e., the transsubcentral gyral approach). The bold circle represents the window made at the subcentral gyrus, and the dashed circle represents the location of the tumor. ALIG = anterior long insular gyrus, ATPG = anterior transverse parietal gyrus, $\mathrm{CS}=$ central sulcus, IPIS = inferior periinsular sulcus, MTG = middle temporal gyrus, $\mathrm{MTPG}=$ middle transverse parietal gyrus, PIP = posterior insular point, PLIG = posterior long insular gyrus, postCG = postcentral gyrus, preCG = precentral gyrus, PTPG = posterior transverse parietal gyrus, SMG = supramarginal gyrus, SPIS = superior periinsular sulcus, $S T G=$ superior temporal gyrus, subCG = subcentral gyrus. Modified with permission from Ture et al: J Neurosurg 90:720-733, 1999.

including his hands, arms, face, mouth, and tongue. The patient did not complain of uncomfortable sensations in the throat or mouth or of epigastric discomfort. Language tasks performed at the same location under stimulation resulted in negative mapping. On the other hand, the medial aspect of the temporal operculum (continuing from the superior temporal gyrus) showed anomia with stimulation (tag 12 in Fig. 3A). Therefore, a cortical incision following $10 \mathrm{~mm}$ of the window was made in the subcentral gyrus for the approach. We then approached by removing the tissue of the subcentral gyrus toward the anterior transverse gyrus, while checking for a negative response following subcortical stimulation. This approach is schematically depicted in Fig. 3A. Subcortical mapping was performed with the same electrical stimulation as used for cortical mapping. At the $20-\mathrm{mm}$ depth, we encountered the tumor tissue, which contained apparent calcified tissue (tissue that was relatively hard and sand-like). A part of the tumor specimen was submitted for frozen sectioning, and the intraoperative pathological diagnosis suggested low-grade glioma. Thus, tumor removal was performed by gyrectomy of the medial part of the anterior transverse parietal gyrus; the gyrus that had been invaded by the tumor was removed until the sulcus bordering the gyri were observed anteriorly and posteriorly. In the deep portion, we observed the surface of the insula and the veins and arteries found above the surface of the insula. The loca- tion we reached was around the posterior insular point, confirmed by the navigation system.

When we stimulated the deep portion of the tumor cavity, specifically the deep medial aspect of the anterior transverse parietal gyrus around the posterior insular point, the patient complained of severe pain in his right side (tag 13 in Fig. 3A). The same response was also observed at the posterior area of the insula just anterior of the posterior insular point (tag 14 in Fig. 3A and D). Although maximum pain was reported in the hip joint, the pain was distributed over almost the entire right side of the patient. After a short rest, we stimulated there again and observed reproducible responses. The patient reported that this pain and discomfort was similar to the symptoms he frequently experienced during his seizures. A summary of intraoperative mapping is shown in Table 2. After confirming that the tumor tissue was completely removed by microscopy, navigation, and intraoperative MRI, general anesthesia was reinduced, cranioplasty was performed with a titanium plate, and the wound was closed. Postoperatively, his seizures have disappeared, with almost 1 year of followup at the time of writing this paper, and the patient shows no neurological deficits, including language and cognitive function (Table 1). No gustatory changes were observed postoperatively. The pathological diagnosis of the tumor was WHO Grade II pleomorphic xanthoastrocytoma. 

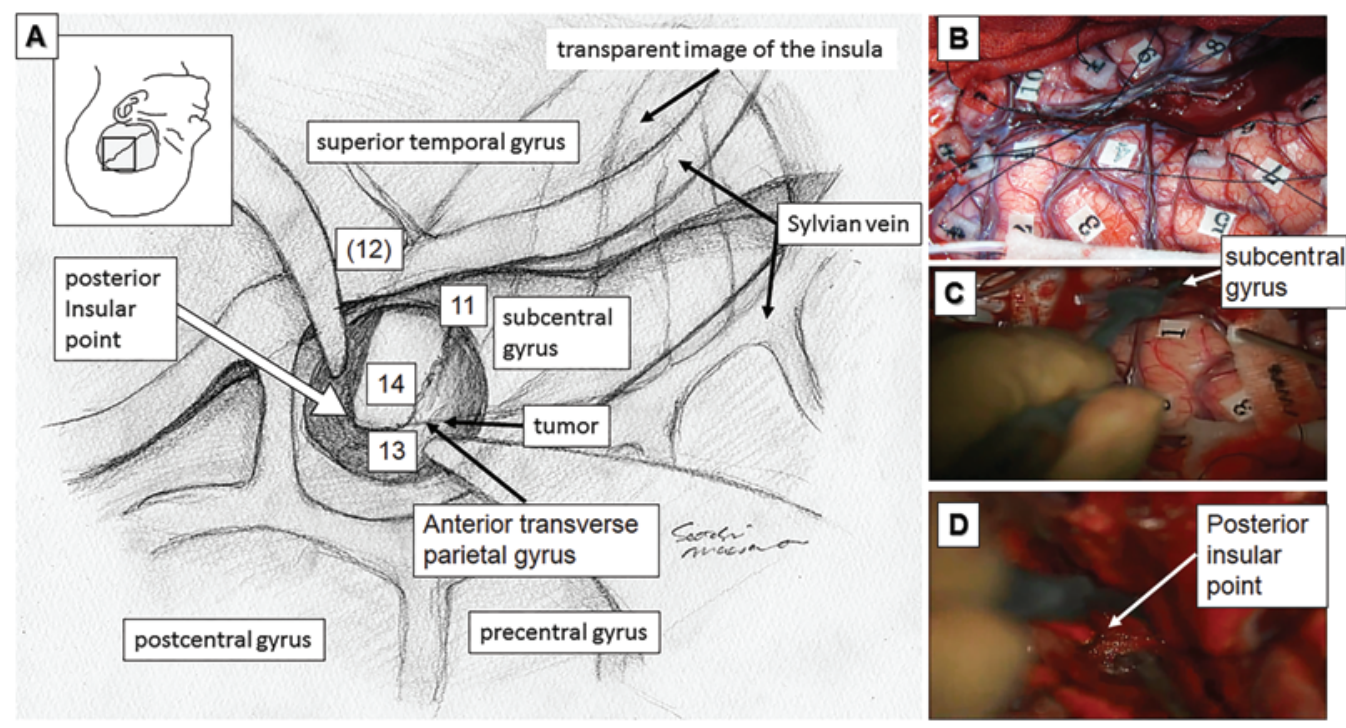

FIG. 3. The appearance of the surgical approach and the intraoperative mapping. A: Schematic diagram of the transcortical approach via the subcentral gyrus. Tags 11-14 show the locations of subcortical stimulation. B: Intraoperative image of the surface of the cortex. Tags 1-10 show the locations of cortical stimulation. C: Image showing the stimulation of the subcentral gyrus. D: Image showing the stimulation of the posterior insula around the posterior insular point. Copyright Satoshi Maesawa. Published with permission.

\section{Discussion}

In this case study, 3 points are clinically important and should be discussed. First, the clinical manifestation of this case was quite rare, but representative of SII epilepsy. Second, the location of the lesion was challenging for surgical removal, and a transcortical approach via the subcentral gyrus, which we performed, was useful. Third, intraoperative mapping demonstrated that the patient experienced pain during the electrical stimulation around the posterior insular point, which was helpful in determining the orientation in this approach.

\section{Secondary Somatosensory Epilepsy}

Characteristics of SII epilepsy include bilateral, wide- spread, and uncomfortable numbness, tingling sensation, and/or occasional pain; this somatotopy is diffuse compared with that of the somatosensory cortex. ${ }^{1,24,35,38}$ Anatomically, the operculoinsular area receives spinothalamic inputs from the thalamic nuclei and has projections via the thalamic nuclei connecting diffusely and bilaterally to the primary somatosensory areas, secondary somatosensory areas, associated somatosensory areas, cingulate gyrus, premotor area, and supplementary motor areas; back-projections from these areas are also received. . $^{8,921,34}$ This connectivity to diffuse areas makes the sensation of epilepsy in this area somatically obscured and symptomatically complicated. In the case presented in this report, the patient experienced uncomfortable dysesthesia in diffuse areas of his body, along with occasional pain; these

TABLE 2. Summary of intraoperative mapping

\begin{tabular}{rlcll}
\hline No. & Anatomical Location & Intensity of Stimulation $(\mathrm{mA})$ & Sensory-Motor Response & Speech Response \\
\hline 1 & Primary sensory cortex & $2-4$ & Dysesthesia in mouth & Delay of speech \\
\hline 2 & Primary sensory cortex & 4 & Dysesthesia in mouth & Delay of speech \\
\hline 3 & Primary motor cortex & 4 & Seizure in rt side of mouth & Speech arrest (motor) \\
\hline 4 & Primary motor cortex & 4 & Tongue movement loss & Delay of speech \\
\hline 5 & Opercular part of IFG & 4 & Negative & Anomia, speech arrest \\
\hline 6 & Triangular part of IFG & 4 & Negative & Anomia \\
\hline 7 & Triangular part of IFG & 4 & Negative & Anomia \\
\hline 8 & STG & 6 & Negative & Semantic aphasia \\
\hline 9 & STG & 6 & Negative & Semantic aphasia \\
\hline 10 & STG & 6 & Negative & Anomia \\
\hline 11 & Subcentral gyrus & $4-6$ & Negative & Negative \\
\hline 12 & Temporal operculum & $4-6$ & Negative & Anomia \\
\hline 13 & ATPG (deep) & 4 & Pain in rt side of body & Negative \\
\hline 14 & Posterior insula & 4 & Pain in rt side of body & Negative \\
\hline
\end{tabular}

ATPG = anterior transverse parietal gyrus; $I F G=$ inferior frontal gyrus; $S T G=$ superior temporal gyrus . 
symptoms were similar to those of SII epilepsy reported in the literature. However, electrophysiological evidence of a symptomatic zone could not be obtained preoperatively in this case. Stereotactic implantation of intracranial electrodes requires passing through the perisylvian cortices, the region that is responsible for language function, i.e., the marginal gyrus, posterior superior temporal gyrus, and clinically important vasculature such as the middle cerebral artery or Sylvian veins. In previous studies, few cases have been studied using intracranial electrode recording, probably because of the anatomical location of the SII area. ${ }^{1,24,36}$ Recently, Isnard et al. reported a case similar to ours that had a lesion (cortical dysplasia) in the right insula and exhibited SII epilepsy; concomitant painful symptoms started from the contralateral hand extending to the whole body (including his head) and intracranial electrode recording confirmed that his seizure started from the lesion. ${ }^{14}$ Although the location of the lesion was somewhat different, i.e., the posterior insula (the medial side to the posterior insular point in their case) and the anterior transverse parietal gyrus (lateral to the posterior insular point in our case), these structures are anatomically contiguous, and symptoms were comparable. Moreover, while our case lacked preoperative electrophysiological examination, considering the symptoms and location of the lesion, his preoperative seizures were believed to represent SII epilepsy.

\section{Transsubcentral Gyral Approach}

The surgical approach for resection of the lesion around the posterior insular point (the posterior insula and the medial aspect of the anterior transverse parietal gyrus) was considerably challenging due to the complicated anatomy, including the critical vasculature near this region, and because this region covered functionally dominant opercular cortices (i.e., the marginal gyrus, angular gyrus, and superior temporal gyrus). No reports have described the details of an approach to resect lesions from such a location. Some reports describing surgery of the insula may be of use as references. ${ }^{67,17,28,37}$ The transsylvian approach with wide opening of the fissure for observation of this location may be effective; however, the risk of vascular injury and pial destruction is not insignificant. Furthermore, the transsylvian approach is associated with high neurological morbidity in surgery for medial temporal tumors. ${ }^{29}$ On the other hand, Sanai et al. and Duffau et al. reported good functional outcomes of surgery for gliomas in the insula using the transcortical approach, i.e., tumor removal via cortical corridors made at the frontal and/or temporal operculum. ${ }^{6,28}$ Sanai et al. achieved $82 \%$ resection for insular gliomas using the transcortical approach, with acceptable postoperative sequelae. Additionally, they insisted that the location of the zone II, superior, and posterior areas of the insula in the Berger-Sanai classification, corresponding to the location of the lesion in our case surrounding the posterior insular point, was especially difficult to access, and that only $67.4 \%$ resection could be achieved in such cases. In our case, cortical mapping of the subcentral gyrus was performed, negative symptoms regarding language and sensory-motor function were confirmed, and a small cortical window was created. Although the location of the pos- terior insular point was further posterior and superior from the subcentral gyrus, the visual angle was not restricted for the surgeons, it was not necessary to retract the parietal operculum, and direct observation of the lesion site was possible. A summary of this approach with schematics and postoperative images is shown in Fig. 4.

In this case, stimulation of the subcentral gyrus caused neither somatosensory nor lingual phenomenon intraoperatively. Gustatory dysfunction was not observed postoperatively. Reports demonstrating electrical stimulation mapping of the subcentral gyrus are rare. Although some authors have found that stimulation in this area may cause some sensory or motor responses and inhibitory responses for the contralateral arm and leg (and occasionally for the ipsilateral limbs), these findings have not been consistent between reports. Additionally, the stimulated sites have not been clearly described (medial or lateral to the Sylvian veins), and conclusions have not been made. ${ }^{35}$ Gustatory function has been shown to be associated with the anterior insula and the frontal operculum in humans, whereas its relationship to more posterior areas, as observed in our case, remains uncertain. ${ }^{27}$ The lack of functional information in the subcentral gyrus may be caused by negative or unclear mapping, which implies that this area does not exhibit a distinct function. Clinical situations may occur in which damage to this area does not cause any deficits. The subcentral gyrus is formed by the fusion of precentral and postcentral gyri in their ventral-most parts and exhibits a U-shaped appearance, potentially representing an anatomically distinct landmark. $22,31,33$

Using this landmark, it may be possible to perform the transcortical approach for reaching the posterior insular region without mapping under awake surgery, although further studies are needed to confirm these findings. Although other approaches may also be possible through the dominant inferior parietal lobule with a combination of cortical mapping ${ }^{19}$ such an approach may result in some undesirable sequelae regarding language function. Therefore, we concluded that the approach taken in this case appeared safe and reasonable for resection of small lesions in and around the posterior insular point of the dominant hemisphere.

\section{Intraoperative Mapping and the Experience of Pain}

In this case, stimulation of the deep portion of the tumor cavity around the posterior insular point (the posterior insula and the medial aspect of the anterior transverse parietal gyrus) caused severe pain in the patient's right side. The findings we obtained here were supported by a report by Mazzola et al. in 2012..$^{20}$ These authors preoperatively evaluated 164 cases of epilepsy using intracerebral electrode stimulation over 12 years and concluded that the medial parietal operculum and posterior insula are the only areas where electrical stimulation is able to trigger activation of the pain cortical network and thus the experience of somatic pain. This region is a part of the "pain matrix," which have been identified as areas that are activated in response to painful stimuli in functional imaging studies, including the insula, the secondary somatosensory cortex located in the suprasylvian parietal operculum, the anterior and midcingulate cortex, the primary somatosensory 

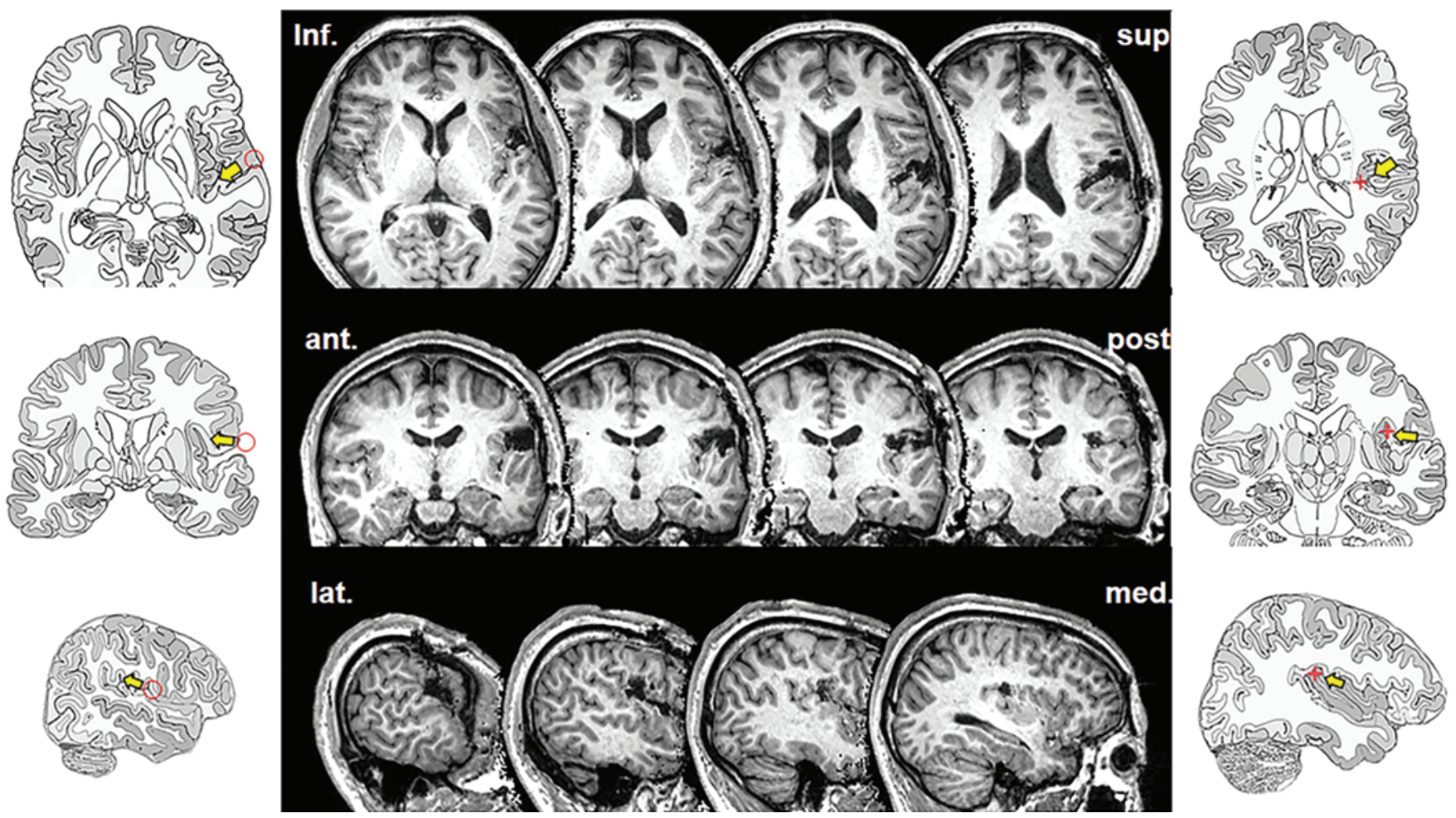

FIG. 4. Multislice postoperative MR images showing the approach via the subcentral gyrus. The red circle represents the location of the cortical window of the subcentral gyrus. The red cross represents the location of the posterior insular point. The yellow arrow represents the direction of the approach. ant. = anterior; Inf. = inferior; lat. = lateral; med. = medial; post $=$ posterior; sup = superior.

cortex, the anterior and frontal cortices, and the primary somatosensory cortex. ${ }^{25}$ However, all other areas (including the midcingulate gyrus) which are also known to play central roles in the experience of pain did not cause pain responses following direct stimulation, and the reason for this remains unclear. ${ }^{13,25,32}$ Thus, in operations near the posterior insular point, pain experience evoked by electrical stimulation could facilitate orientation within the brain tissues. In our case, electrical stimulation of this region reproduced some of his symptoms of epilepsy and supplemented the electrophysiological data, leading to successful tumor resection with achievement of good control of epilepsy.

\section{Acknowledgments}

The authors thank Daisuke Hara and Junko Sugiura (Department of Rehabilitation, Nagoya University Hospital) for evaluation of the language and cognitive function of the patient. This study was supported by a Grant-in-Aid for Scientific Research (KAKENHI, no. 26462202).

\section{References}

1. Blume WT, Jones DC, Young GB, Girvin JP, McLachlan RS: Seizures involving secondary sensory and related areas. Brain 115:1509-1520, 1992

2. Burton H, Abend NS, MacLeod AM, Sinclair RJ, Snyder AZ, Raichle ME: Tactile attention tasks enhance activation in somatosensory regions of parietal cortex: a positron emission tomography study. Cereb Cortex 9:662-674, 1999

3. Casey KL, Morrow TJ, Lorenz J, Minoshima S: Temporal and spatial dynamics of human forebrain activity during heat pain: analysis by positron emission tomography. J Neurophysiol 85:951-959, 2001

4. Davis KD, Pope GE, Crawley AP, Mikulis DJ: Neural correlates of prickle sensation: a percept-related fMRI study. Nat Neurosci 5:1121-1122, 2002

5. Diamond ME, Harris JA, Petersen RS: Sensory learning and the brain's body map, in Nelson RJ (ed): The Somatosensory System. Boca Raton, FL: CRC Press, 2002, pp 183-195

6. Duffau H: A personal consecutive series of surgically treated 51 cases of insular WHO Grade II glioma: advances and limitations. J Neurosurg 110:696-708, 2009

7. Duffau H, Moritz-Gasser S, Gatignol P: Functional outcome after language mapping for insular World Health Organization Grade II gliomas in the dominant hemisphere: experience with 24 patients. Neurosurg Focus 27(2):E7, 2009

8. Eickhoff SB, Amunts K, Mohlberg H, Zilles K: The human parietal operculum. II. Stereotaxic maps and correlation with functional imaging results. Cereb Cortex 16:268-279, 2006

9. Eickhoff SB, Schleicher A, Zilles K, Amunts K: The human parietal operculum. I. Cytoarchitectonic mapping of subdivisions. Cereb Cortex 16:254-267, 2006

10. Fujii M, Maesawa S, Motomura K, Futamura M, Hayashi Y, Koba I, et al: Intraoperative subcortical mapping of a language-associated deep frontal tract connecting the superior frontal gyrus to Broca's area in the dominant hemisphere of patients with glioma. J Neurosurg 122:1390-1396, 2015

11. Hämäläinen H, Hiltunen J, Titievskaja I: Activation of somatosensory cortical areas varies with attentional state: an fMRI study. Behav Brain Res 135:159-165, 2002

12. Hari R, Karhu J, Hämäläinen M, Knuutila J, Salonen O, Sams $\mathrm{M}$, et al: Functional organization of the human first and second somatosensory cortices: a neuromagnetic study. Eur J Neurosci 5:724-734, 1993 
13. Hutchison WD, Davis KD, Lozano AM, Tasker RR, Dostrovsky JO: Pain-related neurons in the human cingulate cortex. Nat Neurosci 2:403-405, 1999

14. Isnard J, Magnin M, Jung J, Mauguière F, Garcia-Larrea L: Does the insula tell our brain that we are in pain? Pain 152:946-951, 2011

15. Kakigi R, Hoshiyama M, Shimojo M, Naka D, Yamasaki $\mathrm{H}$, Watanabe $\mathrm{S}$, et al: The somatosensory evoked magnetic fields. Prog Neurobiol 61:495-523, 2000

16. Kayama T: The guidelines for awake craniotomy guidelines committee of the Japan awake surgery conference. Neurol Med Chir (Tokyo) 52:119-141, 2012

17. Lang FF, Olansen NE, DeMonte F, Gokaslan ZL, Holland EC, Kalhorn C, et al: Surgical resection of intrinsic insular tumors: complication avoidance. J Neurosurg 95:638-650, 2001

18. Maesawa S, Fujii M, Nakahara N, Watanabe T, Wakabayashi $\mathrm{T}$, Yoshida J: Intraoperative tractography and motor evoked potential (MEP) monitoring in surgery for gliomas around the corticospinal tract. World Neurosurg 74:153-161, 2010

19. Maldonado IL, Moritz-Gasser S, de Champfleur NM, Bertram L, Moulinié G, Duffau H: Surgery for gliomas involving the left inferior parietal lobule: new insights into the functional anatomy provided by stimulation mapping in awake patients. J Neurosurg 115:770-779, 2011

20. Mazzola L, Isnard J, Peyron R, Mauguière F: Stimulation of the human cortex and the experience of pain: Wilder Penfield's observations revisited. Brain 135:631-640, 2012

21. Mufson EJ, Mesulam MM: Thalamic connections of the insula in the rhesus monkey and comments on the paralimbic connectivity of the medial pulvinar nucleus. J Comp Neurol 227:109-120, 1984

22. Naidich TP, Valavanis AG, Kubik S: Anatomic relationships along the low-middle convexity: Part I-Normal specimens and magnetic resonance imaging. Neurosurgery 36:517-532, 1995

23. Ojemann G, Ojemann J, Lettich E, Berger M: Cortical language localization in left, dominant hemisphere. An electrical stimulation mapping investigation in 117 patients. J Neurosurg 71:316-326, 1989

24. Penfield W, Jasper H: Epilepsy and Functional Anatomy of the Human Brain. Boston: Little, Brown, 1954

25. Peyron R, Laurent B, García-Larrea L: Functional imaging of brain responses to pain. A review and meta-analysis (2000). Neurophysiol Clin 30:263-288, 2000

26. Ploner M, Schmitz F, Freund HJ, Schnitzler A: Parallel activation of primary and secondary somatosensory cortices in human pain processing. J Neurophysiol 81:3100-3104, 1999

27. Purves D, Augustine GJ, Fitzpatrick D, Katz LC, LaMantia AS, McNamara JO, et al (eds): Neuroscience, ed 2. Sunderland, MA: Sinauer Associates, 2001

28. Sanai N, Polley MY, Berger MS: Insular glioma resection: assessment of patient morbidity, survival, and tumor progression. J Neurosurg 112:1-9, 2010
29. Schramm J, Aliashkevich AF: Surgery for temporal mediobasal tumors: experience based on a series of 235 patients. Neurosurgery 60:285-295, 2007

30. Standard Language Test of Aphasia Committee: Standard Language Test of Aphasia: Manual of Directions, ed 2. Tokyo: Homeido, 1977

31. Türe U, Yaşargil DC, Al-Mefty O, Yaşargil MG: Topographic anatomy of the insular region. J Neurosurg 90:720-733, 1999

32. Vogt BA: Pain and emotion interactions in subregions of the cingulate gyrus. Nat Rev Neurosci 6:533-544, 2005

33. Wagner M, Jurcoane A, Hattingen E: The U sign: tenth landmark to the central region on brain surface reformatted MR imaging. AJNR Am J Neuroradiol 34:323-326, 2013

34. Weiss N, Lawson C, Greenspan J, Ohara S, Lenz FA: Studies of the human ascending pain pathways. Thalamus Relat Syst 3:71-86, 2005

35. Woolsey CN, Erickson TC, Gilson WE: Localization in somatic sensory and motor areas of human cerebral cortex as determined by direct recording of evoked potentials and electrical stimulation. J Neurosurg 51:476-506, 1979

36. Yamamoto J, Ikeda A, Matsuhashi M, Satow T, Takayama M, Ohara S, et al: Seizures arising from the inferior parietal lobule can show ictal semiology of the second sensory seizure (SII seizure). J Neurol Neurosurg Psychiatry 74:367-369, 2003

37. Yaşargil MG: Microneurosurgery. New York: Thieme Medical, 1996

38. Young GB, Barr HW, Blume WT: Painful epileptic seizures involving the second sensory area. Ann Neurol 19:412, 1986 (Letter)

\section{Disclosure}

The authors report no conflict of interest concerning the materials or methods used in this study or the findings specified in this paper.

\section{Author Contributions}

Conception and design: Maesawa, Fujii, Futamura, Wakabayashi. Acquisition of data: Maesawa, Fujii, Futamura, Hayashi, Iijima. Analysis and interpretation of data: Maesawa, Fujii, Futamura. Drafting the article: Maesawa. Critically revising the article: Maesawa, Fujii, Futamura. Reviewed submitted version of manuscript: Maesawa, Fujii. Approved the final version of the manuscript on behalf of all authors: Maesawa. Administrative/ technical/material support: Hayashi, Iijima. Study supervision: Wakabayashi.

\section{Correspondence}

Satoshi Maesawa, 65 Tsurumai, Showa, Nagoya 466-8550, Japan. email: smaesawa@med.nagoya-u.ac.jp. 